\title{
METHOD AND THE MACHINE FOR TRAINING THE RUNNING SPEED
}

\section{Tomina-Dana PETRESCU, Dragoș IONESCU BONDOC}

\begin{abstract}
Former sprint runner, I was particularly concerned with sprint training methodology. In this regard, I have experimented together with our training colleagues, an exercise that stimulates the increase of speed of the steps in speed running, using an installation made by our own coach, designed to stimulate and increase the frequency of the steps, coaching the so-called "super-speed". In order to increase the running speed, besides developing motor skills, a series of technical objectives must be achieved through training.

It is known that using run-on exercises, similar to running on a downhill slope, there is a series of positive influences on the kinetics of the running speed of which the impulse angle and the orientation of the impulse vector in the CGM on - the impulse angle is primordial, along with:

-achieving the optimum ratio between the frequency and the length of the steps;

-increasing the power released by the impulse foot;

-decreasing the impulse angle;

-increasing the frequency of the treadmills achieved per unit of time;

-increasing the length of the steps.

Our experiment has attempted to justify this kind of exercise in speed training. Finally, it was found that this exercise positively affects the last two goals listed above.
\end{abstract}

Keywords:super-maximal speed, impulse angle, optimal ratio of the frequency of the steps and the length of steps.

\section{Introduction}

The paper entitled Method and device for training the running speed, shows an exercise, maybe the most efficient, for the development of running speed which breaks up an automatism that occurs in all sprinters immediately after they have stabilized in terms of morphological growth known as the speed barrier.

\section{Problem Statement}

To break this automatism, which appears after the completion of growth, stimulus is needed to stimulate the shortening of alternation between CNS excitation and inhibition, using exercises that can be performed with the same amplitude at higher speeds than the athlete can do in normal running. The lack of exercises leading to the value increase of the performances of the Romanian sprinters, which made Romanian sprint no longer reflected among participants in major competitions. The most used are downhill running on a slight slope, but it has the disadvantage that at some point the accumulated speed exceeds the possibilities of controlling contact with the ground "by arresting braking", or using the back winding "if they have it".

Starting with the problems presented above, we experimented with our training colleagues a towing method with a device designed to achieve constant thrust at slightly higher speeds than we did in free running since 2002 .
These towing exercises are most effective for developping because they produce "stronger and more active stimuli that will cause appropriate psychological and physical changes." (Bompa, T. 1999)

\section{Research Questions/Aims of the research}

The paper aims to present an original way to trainrunning speed, by a method that is perhaps the first of its kind in Romania, and not only, because at the time when his device was built, such modalities were not known in training the running speed.

Starting from the hypothesis of those shown above, the study started from using the training exercises in the unloading exercise to increase the frequency of the running steps on a unit of time, while increasing the length of the steps.

The running speed depends on the principles of biomechanics of the running step, the most important of which are the angle formed by the vector of the surface of the running pulse frequency and the rate (steps per second).

The solution can come from the most effective exercises that can solve problems such as: "breaking the barrier" to increase the frequency of the steps, reducing the "braking force" by bringing the contact with the ground as close as possible to the C.M. (center of mass) on the ground, plowing the pelvis and the knee of the 
pendulum foot into a greater flexion, and the thigh horizontally forward.

\section{Research Methods}

In order to solve the problems specific to the running speed described above, there has been designed a towing device with adjustable speeds in accordance with the possibilities of the athlete. To be effective, it must ensure the following conditions:

- to faithfully reproduce the running speed as closely as possible to the training and competition, but with a lower level of force demand compared to normal use;

- to allow for a higher frequency than that which it performs in normal running;

- unloading the run request to be uniformly accelerated without shocks when the device is engaged;
- It can be quickly disconnected in cases of emergency (muscle contractures and others);

- a constant supermaximal speed - between $0.5 \mathrm{~m} / \mathrm{s}$ $-1 \mathrm{~m} / \mathrm{s}$, in order not to generate brakes in contact with the ground;

- to ensure constant discharge over a distance of 40 $\mathrm{m}-60 \mathrm{~m}$.

\section{Findings}

Trailer running. Schematically the exercise would be as in Figure 1.

The research was conducted over five weeks when the outdoor season began, the subject is an athlete - a senior - who in the past two years failed to run on more than $200 \mathrm{~m} 25.23 \mathrm{sec}$.

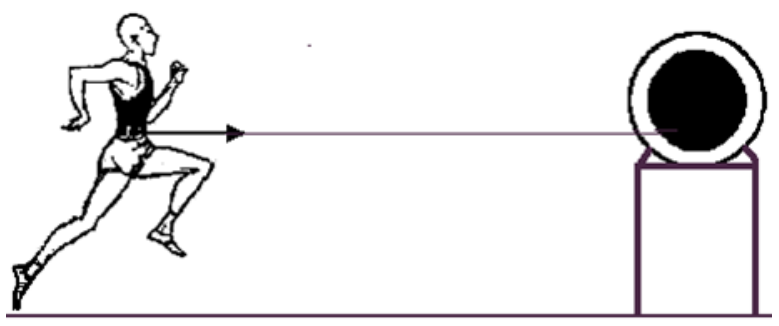

Fig. 1. Scheme on the workings of the unit "Tomi Sprint" and how they act on the pelvis traction wire athlete while running

Coming after the competition the indoor season, the preparation was at fairly good parameters, it was also possible to work for the "supervision" training, trying to keep the training at higher parameters.

We have also have the data obtained from the analysis of video recordings before and after 15 workouts outdoors which led to a series of values that showed a substantial progression of steps running at the end of the study. These progressions have been reflected.
Initial recordings were made using the "superspeed" training system, of which we show in Figure 2A, the impulse completion during the first training and the same phase of impulse completion after 5 weeks of towing work in "super-speed" conditions.

Taking into account that the recordings were made with a video camera with 25 frames per second (i.e., 0.04 sec / frame), the results recorded in Table 1 show a maximum error of $0.03 \mathrm{sec}$, more than acceptable in from the point of view of the scientific research.

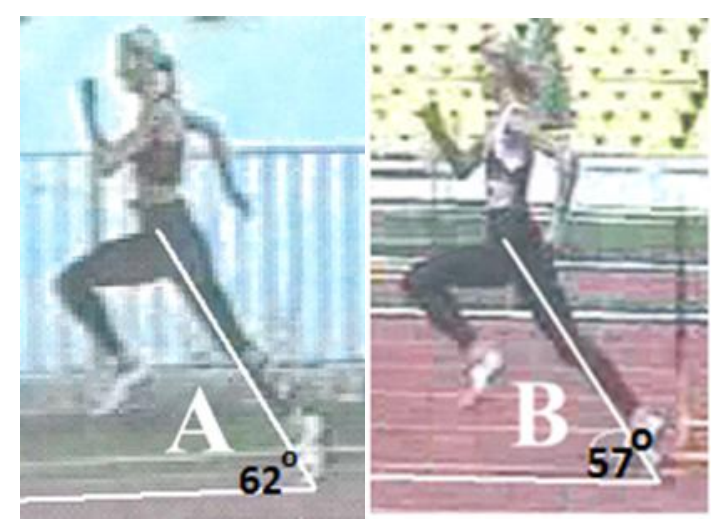

Fig. 2. The impulse angle generated by the runner in the initial test (A) and the final test (B). 
From the records we took into consideration two identical sequences from the initial and final evolutions, which corresponded almost identically from the point of view of the distances and the shooting angles, based on which the angles of the segments can be highlighted by a very small error in the angle that the impulse vector passing through the $\mathrm{CM}$ of the runner with the running surface (horizontal) makes. Although the difference can also be seen with the naked eye, measuring them we found an angle of about $62^{\circ}$ for the initial test (A) and about $57^{\circ}$ for the final test (B).

From the positive effects of this exercise also benefited runner Carmen Zamfir, who after only 3 workouts in 2 weeks, achieved an improvement in performance from $8.32 \mathrm{sec}$ to $7.96 \mathrm{sec}$ on $60 \mathrm{mg}$, at the Indoor European Championship, Stefan Adrian, who after only five training progressed from 10.20 seconds to 10.36 seconds per $100 \mathrm{~m}$, MitoiuAdriam who after 4 training progressed from 7.46 seconds to 7.01 seconds on $60 \mathrm{~m}$, 2nd place at National Championship for Jr. I.

\section{Discussions}

By biomechanically analyzing the two sequences shown in FIG. 2 we can also see the difference between the forward pendulum amplitude in sequence $2 \mathrm{~B}$, the work of the calf acting in the knee joint is increased, increasing the pendulum inertia.
An altered evolution for the better also had the work of the arms, which, as seen in Fig. 2A, the vertical plane of the hand exceeds the vertical flank of the elbow generating a negative inertia towards the back. Finally, we can observe that the work of the arms improved.

\section{Conclusions}

Following the experiment, there were some changes to the running technique of the running step without any other intervention in training such as:

- decreasing the impulse angle at the end of the research by about 5 degrees;

- the pendulum leg with the hip-thigh close to the horizontal that trains the basin;

- at the end of the impulse, the vertical plane of the arm does not extend backwards the vertical plane of the elbow, generating a perpendicular vertical inertia.

\section{References}

[1] Dintiman, G. B; Ward, R. D. 1988 Sport Speed - in Program on how to improve speed; SDP 410-412

[2] Gagea A; - 2006 ; Analytical biomechanics. Ed. Char. Dounias \& Co. Greece.

[3] Legros, L.; - 1980. La biochimie au service du sprinter. Sport Belgique.

[4] Petrescu, T.; Petrescu,T.,D.;2017;Development of running speed, Editura pentru sport; București. 